\title{
How to build and sustain an academic cardiothoracic surgery program
}

Leora T. Yarboro, MD, J. Hunter Mehaffey, MD, MSc, Eric J. Charles, MD, PhD, and Irving L. Kron, MD

Feature Editor's Introduction-The Holy Grail of academic surgery programs is to have meaningful influence. That sustained success over time leads to positive changes in patients' lives, our profession, and on the science of surgery. It is with that as a backdrop that Yarboro and colleagues give us their perspectives on the foundational principles of the sustained success of the University of Virginia cardiac and thoracic surgery programs.

In their article, we see a framework set in place by $\mathrm{Dr}$ Kron of excellence in clinical care, education, research, diversity, and mentorship. Although these are not novel pillars of academia, the translation into practice is the real accomplishment. To educate at the highest level-and maintain outstanding outcomes-the teachers themselves must have a firm grasp on content and technique. The provision of protected time and mentorship for research is required to establish an independent career. The multifaceted leadership and institutional awareness requisite to garner support and resources are essential in facilitating a faculty that flourishes in an increasingly fiscally constrained environment.

With a mixture of hard work, perseverance, vision, and certainly luck, this group has been successful in academia over the course of decades. Not only were the goals accomplished at a point in time, but also through dedicated mentorship, these values were instilled into subsequent generations over decades. Much akin to quotation attributed to Aristotle, "We are what we repeatedly do. Excellence, then, is not an act, but a habit," the mantra ascribed is of continued excellence. Is this not impact then?

\section{Bryan A. Whitson, MD, PhD, and Nahush A. Mokadam, MD}

Thirty years ago, our program in cardiothoracic surgery was a clinically focused enterprise with graduates of the training program largely pursuing private practice. Then came a conscious decision by the senior leadership-including

From the Division of Cardiac Surgery, Department of Surgery, University of Virginia, Charlottesville, Va.

Received for publication Jan 5, 2021; revisions received March 14, 2021; accepted for publication March 26, 2021; available ahead of print May 13, 2021.

Address for reprints: Leora T. Yarboro, MD, Department of Surgery, University of Virginia, PO Box 800679, Charlottesville, VA 22908 (E-mail: LJT9R@hscmail. mcc.virginia.edu).

J Thorac Cardiovasc Surg 2022;163:1431-4 0022-5223/\$36.00

Copyright (c) 2021 Published by Elsevier Inc. on behalf of The American Association for Thoracic Surgery

https://doi.org/10.1016/j.jtcvs.2021.03.129

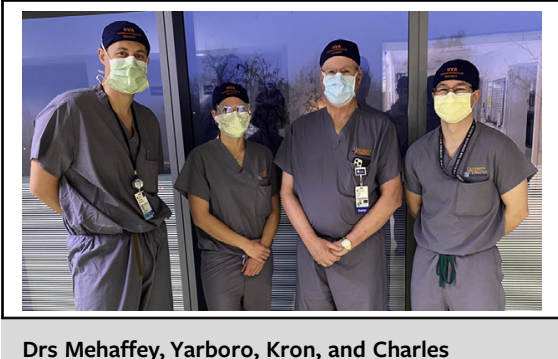

Drs Mehaffey, Yarboro, Kron, and Charles

CENTRAL MESSAGE

Five interrelated components

that are key to building and sus-

taining a successful academic

cardiothoracic surgery program

include excellence in clinical,

teaching, research, diversity, and

mentorship.

See Commentary on page 1435.

the senior author of this article-to build a program that was not just strong clinically but that would ultimately develop future academic cardiothoracic surgeons. The charge required a paradigm shift that touched all levels, from recruitment to research and education. Over the past quarter century, through intentional and iterative processes, we believe it is safe to say that we have been able to realize our goal. The challenge we have before us now is to sustain the objectives of an academic surgery program at a time when a hyperanalytic focus on performance metrics attempts to define our very beings as cardiothoracic surgeons. Education and research, cornerstones of our academic surgery program, are threatened as clinical demand increases and resources become scarce.

It is within the context of the history of our own institution and our pursuit to sustain and grow, that we reflect and attempt to describe 5 key components that we believe have been central to our program's success. These include clinical excellence, teaching, research, diversity, and mentorship.

\section{CLINICAL EXCELLENCE}

Surgeons recognize that patient outcomes define the success of our medical practice. The ability to select and perform the appropriate operation for each patient is 
fundamental. We also understand that as patient and procedural complexity increase, so too does the demand on perioperative care. To this end, academic medical centers often function as referral sites for other regional hospitals, either because there is a unique technique or technology that is being offered or because patient complexity exceeds resources available at the local center.

For us to offer the next level of care, it is important that we work cohesively with our referring physicians as well as with colleagues within our own institution. We were early adopters of the multidisciplinary team approach to patient care. Our belief is that a good multidisciplinary team is accessible, maintains expertise on all fronts, and practices an openness of thought and respect in communication. The context of our work within an academic medical center means that we also track and share outcomes data to inform best practice. We are constantly querying our results and completing programmatic comparisons to ensure that we are holding ourselves to the highest standards. Indeed, with this process as our bedrock we have been able to attract numerous device and medical trials, which, in turn, bolsters our distinction and increases our ability to treat people who would otherwise be turned down for advanced care.

The clinical enterprise highlighted here is important and there is no doubt that working within a multidisciplinary team to inform future practice is extremely rewarding. However, as the business of medicine becomes more overt within academic medical centers, we have noticed a shift away from our core in terms of how we are structured within the hospital system. Historically, cardiothoracic surgeons were integrated within the Department of Surgery as a nod to a common training pathway. Now, each academic institution seems to have a variable approach to how surgeons are grouped. In June 2016, we split from a unified thoracic, cardiac, and vascular practice into 3 individual divisions still within the Department of Surgery with the cardiac division also including congenital. Hospital service lines such as oncology, heart and vascular, and pediatrics cut across these divisions, attempting to streamline patient care based on disease pathology. To maintain the culture of our academic program and align our research and educational efforts, the divisions of cardiac throracic and vascular surgery continue to meet on a monthly basis.

How to navigate the ever-changing waters of academic medicine can be complicated and each center will find its own path. It is important for cardiothoracic surgeons to maintain a proactive stance within their institution. It is only through engagement and advocacy that we are able to maintain the original objective, which is to provide exceptional and individualized care for our patients.

\section{EDUCATION}

Many will question whether one can achieve excellent clinical outcomes while training the next generation of cardiothoracic surgeons. Indeed, this is the challenge posed to and readily accepted by surgical educators. Teaching procedures that are both complex and time sensitive is the next level of operating and requires commitment and focus. In turn, there is no greater reward than watching the next generation of cardiothoracic surgeons succeed as they transition into practice.

There are certain fundamentals that all successful academic cardiothoracic training programs will have. The first has already been mentioned: Faculty must be wholly committed to the process of education. Supervision and constant feedback are critical as educators "shift to the left" as Tribble describes in his 2017 editorial on resident training. ${ }^{1}$ At no point is patient care compromised. Additionally, the use of adjunct teaching formats, including surgical simulation, didactic conferences, mock orals, and journal clubs helps ensure that fundamental skills and fund of knowledge are achieved. Educating resident physicians takes time beyond that of clinical care. This is a commitment that needs to be fostered and supported within the institution. It is also a commitment that we are happy to make because each year we get to see a group of incredibly bright and inspired individuals learn the nuances of our field.

There are currently 3 distinct training paradigms in cardiothoracic surgery. These include the integrated-6, 4-3 as well as the traditional 2- or 3-year fellowship. Each training program is as unique as its constituents and it is important to understand that what works for 1 institution may not be appropriate for another. We graduate 3 residents per year and have had all 3 training tracks represented at our institution since 2011. We have learned over the years to be flexible with the curriculum and accommodate the needs of the learner. We also, through years of training, have an understanding of what we believe the finished product should look like. No matter what training program a resident comes through, we push to that mold.

Successfully transitioning our resident physicians into practice during their final years of training requires a particular dedication from faculty in the operating room. Nowhere else is the trainee going to be able to fully integrate the skills he or she has learned with the nuances of tissue handling, judgment, and leadership that are essential components of performing safe cardiothoracic surgery. To achieve this, there must be confidence on the side of the educator as well as a deep understanding of the technical abilities of the learner. Progression to the next step is an active process for both the educator and the trainee. Although it might look like a leap of faith to have resident physicians perform critical steps in a cardiac operation, surgical educators know that this is, in fact, the culmination of years of practice and preparation.

Overall, the cardiothoracic training program has been a hallmark of our program. The dynamic culture of education 
drives recruitment of the next generation of outstanding trainees committed to learning our trade. Our belief is that great recruits make the program and you get the best resident physicians if they know they are going to learn how to operate. Therefore, we continue to commit to the process and are rewarded each year with a graduating class that represents the future of our profession.

\section{RESEARCH}

The opportunity to perform research that furthers the field of cardiothoracic surgery is among the most rewarding aspects of academic medicine. However, it can be difficult for surgeons to integrate research effectively. This is particularly true if the clinical enterprise is preferentially valued. Maintaining a successful research program therefore requires an intentional and multifaceted approach.

We are fortunate that we now have a long history of successful research and funding; however, this was not a guarantee starting out. On our first application to the National Institutes of Health (NIH) for a cardiothoracic surgery training grant, the pink sheet proclaimed "Surgeons should be in the [operating room] so the rest of us can do research." Hard to imagine a more deflating response, but that statement was the motivation for ultimately successfully obtaining funding in the form of an NIH T32 grant and sustaining it for a quarter century. ${ }^{2}$

Surgeons are invaluable members of the team when it comes to recognizing and addressing the unique needs of our patient populations. It is also part of our charge to inspire that belief in the next generation of surgeons. To that end, much of our success in research stems from promotion of our resident physicians and junior faculty. All residents in the integrated-6 and 4-3 programs spend at least 2 years dedicated to research during their training. They are supported in their efforts by cardiothoracic surgery faculty who block time to engage in laboratory and outcomes research meetings. Protecting this time is essential and has been extremely fruitful for residents and faculty alike. We also suspend all elective clinical activities during our annual Resident Research Day to focus on sharing and celebrating the research being performed.

Our junior faculty members also benefit from a structured research program. They are provided a stipend for a research assistant and work with a mentor, typically outside our department, until they are ready to become independent. The aim is $40 \%$ to $50 \%$ protected research time for first 3 years to provide a favorable environment to achieve extramural funding. We have focused on supporting faculty with departmental funds, statistical support, and grant management assistance to further their research. Finally, we have developed a research incentive program using an assessment tool to financially reward academic productivity the same way we financially reward clinical productivity. ${ }^{3}$
These efforts have resulted in 4 out of 11 of our cardiothoracic faculty members having extramural funding as of the beginning of this academic year.

Over the years, our research efforts have been augmented successfully through collaborative efforts that extend beyond our own institution. We have learned a tremendous amount through active participation in national societies as well as through database research. Two efforts to highlight include our participation with Virginia Cardiac Services Quality Initiative and the NIH as part of the Cardiothoracic Surgery Trials Network. The Virginia Cardiac Services Quality Initiative is a consortium of private and academic hospitals across the state of Virginia who came together in the mid-1990s with a common goal to improve quality for our patients. Through this collaborative, we share data and use collective expertise to drive quality improvement, health care policy, and economics for patients in our region. ${ }^{4,5}$ The Cardiothoracic Surgery Trials Network has conducted numerous surgery-related clinical trials to develop evidence-based guidelines for the practice of cardiac surgery. ${ }^{6}$ Work within this network has enabled us to perform research that influences at a national level.

We believe our efforts to grow a vibrant research program to be generalizable with the appropriate commitment from faculty and buy-in at the level of the institution. Indeed, other centers have highlighted success with research protocols and engagement programs. ${ }^{7,8}$ Ultimately, if the culture and expectation of academic pursuit exists, then productivity and achievement will grow exponentially. Finally, when prospective patients see your academic publications online, it provides an additional boost to clinical volume as they seek out your expertise.

\section{DIVERSITY}

Achieving a successful academic cardiothoracic surgery program requires diverse ideas, backgrounds, and approaches. It is with this foundation that we recognize and address the myriad of barriers that affect our patients' care. How do you get to that point where everyone buys in? Emphasize it. Make it the culture. Everyone in the department needs to have a clear understanding of the mission and believe that he or she helps support it. By intentionally recruiting and promoting diversity, we have achieved some success: Nearly half of the trainees in our department over the past 10 years are women or underrepresented in medicine individuals. Additionally, we are among the few departments to have female leaders as division chiefs of both cardiac surgery and thoracic surgery.

Of course diversity extends beyond the physical and a well-balanced faculty has additive but varied interests and represents a range of expertise. It is important that each member of the team see a clear lane in which they can grow. Our team has been relatively small, fluctuating 
between 3 and 6 members in adult cardiac surgery over the past 10 years. For us, what has worked is achieving a balance between clinical expertise and competency. For instance, we recognize that if a surgeon becomes expert at a particular procedure to the point of exclusivity, then the group will likely need to be larger and may risk practice gaps if there is attrition. On the other hand, if there is baseline competency maintained across the group and redundancy within the program clinically, then there can be more flexibility for team members to explore research or other professional goals.

Creating diversity within a group is an important process that, when done well, has the ability to unlock potential. Maintaining diversity is equally important and is an active process that requires objective evaluation of each new recruit, hire, and idea. When recruiting, there will be candidates who are exceptional, both on the trainee and faculty level. It is important to recongize, however, that "fit" is not as much a function of skill set as it is mentality. When trainees within the system fit the mission, it is resonable to recruit internally to keep the best people. A recent article by Bajaj and colleagues ${ }^{9}$ examining the topic of hiring trainees into the same institution found that individuals were more academically productive with similar career advancement.

\section{MENTORSHIP}

The glue that keeps a program together and functioning cohesively is mentorship. Indeed, the bond that is achieved during training is something to be respected and cherished. We believe, as academic surgeons, that mentorship is not limited to a moment in time or place. ${ }^{10}$ Sponsorship and promotion of a mentee continues across the span of a career. Maintaining these bonds is essential because it enables cardiothoracic surgeons to remember their roots and creates opportunities for future trainees. Ultimately, the measure of our success as an academic program is evidenced by more than $70 \%$ of our trainees going into academic practice, including 14 division chiefs and 4 departmental chairs over the past 25 years. We are able to stay connected as this tight-knit group through an email listserv that contains all graduates and faculty at the University of Virginia. Using this forum, we continue to learn from each other, provide mentorship, and network for job opportunities.

\section{CONCLUSIONS}

The culture we have been able to create and cultivate now spans generations, making it resilient and self-defining. The value in this pursuit speaks for itself: new knowledge, expert clinical outcomes, and the next generation of academic cardiothoracic surgeons. Ultimately, different institutions have different challenges, but through a similar framework this approach should be possible anywhere.

\section{Conflict of Interest Statement}

Dr Yarboro is proctor and consultant for Medtronic. All other authors reported no conflicts of interest.

The Journal policy requires editors and reviewers to disclose conflicts of interest and to decline handling or reviewing manuscripts for which they may have a conflict of interest. The editors and reviewers of this article have no conflicts of interest.

\section{References}

1. Tribble Md C. A shift to the left: teaching the craft of cardiac surgery. Heart Surg Forum. 2017;20:E269-73.

2. Narahari AK, Charles EJ, Mehaffey JH, Hawkins RB, Schubert SA, Tribble CG, et al. Cardiothoracic surgery training grants provide protected research time vital to the development of academic surgeons. J Thorac Cardiovasc Surg. 2018;155:2050-6.

3. Schroen AT, Thielen MJ, Turrentine FE, Kron IL, Slingluff CL Jr. Research incentive program for clinical surgical faculty associated with increases in research productivity. J Thorac Cardiovasc Surg. 2012;144:1003-9.

4. Mehaffey JH, Hawkins RB, Byler M, Charles EJ, Fonner C, Kron I, et al. Cost of individual complications following coronary artery bypass grafting. $J$ Thorac Cardiovasc Surg. 2018;155:875-82.e871.

5. Hawkins RB, Mehaffey JH, Yount KW, Yarboro LT, Fonner C, Kron IL, et al. Coronary artery bypass grafting bundled payment proposal will have significant financial impact on hospitals. J Thorac Cardiovasc Surg. 2018;15:182-8.

6. Kron IL, LaPar DJ, Horvath KA; CTSN Investigators and The American Association for Thoracic Surgery Scientific Affairs and Government Relations Committee. Cardiothoracic surgical trials network: evidence-based surgery. J Thorac Cardiovasc Surg. 2016;151:28-9.

7. Coyan GN, Sultan I, Seese LM, Chu D, Schuchert MJ, Kinnunen A, et al. Implementation of a protocol to increase the academic productivity of cardiothoracic surgery resident physicians. J Thorac Cardiovasc Surg. October 7, 2020 [Epub ahead of print].

8. Frankel WC, Scott BG, Massarweh NN, Silberfein EJ, Zhang Q, Rosengart TK, et al. A multifaceted research engagement program improved the academic productivity of general surgery residents. J Surg Educ. 2020;77:1082-7.

9. Bajaj SS, Wang H, Williams KM, Pickering JM, Heiler JC, Manjunatha K, et al New attending surgeons hired by their training institution exhibit greater research productivity. Ann Thorac Surg. November 2, 2020 [Epub ahead of print].

10. Kron IL. Surgical mentorship. J Thorac Cardiovasc Surg. 2011;142:489-92.

Key Words: academic cardiothoracic surgery, cardiothoracic surgery education, cardiothoracic surgery research, cardiothoracic surgery 